\title{
Localization of Tactile Stimuli Depends on Conscious Detection
}

\author{
Justin A. Harris, Lisa Karlov, and Colin W. G. Clifford \\ School of Psychology, University of Sydney, Sydney 2006, Australia
}

\begin{abstract}
Neurological reports of "tactile blindsight" suggest that the human somatosensory system can extract behaviorally useful information about the location of a tactile stimulus in the absence of conscious awareness that the stimulus occurred (Paillard et al., 1983; Rossetti et al., 1995). However, in a series of psychophysical experiments with neurologically intact subjects, we found no evidence for such a dissociation. Our subjects' ability to name the finger on which a tactile stimulus had been presented was dependent on their ability to consciously detect that stimulus (Harris et al., 2004). The present experiments followed up on this study and specifically sought evidence for a dissociation when subjects were required to indicate the location of the stimulus either by pointing at or moving the stimulated finger, the same response made by the neurological patients. Once again, localization accuracy was correlated with detection, and, crucially, when both detection and localization were measured using equivalent forced-choice tasks, the subjects were completely unable to identify the location of stimuli that they had not detected. These findings are inconsistent with the dissociation implied by the cases of tactile blindsight, but are consistent with other neurological evidence that detection of a tactile stimulus does not depend on localization (Head and Holmes, 1911; Halligan et al., 1995; Rapp et al., 2002).
\end{abstract}

Key words: somatosensory; touch; blindsight; masking; vibrotactile; consciousness

\section{Introduction}

The last decade has seen a ground-swell of research directed at identifying the neural correlates of conscious perception. Much of this interest has its roots in studies of the neuropsychological syndrome "blindsight," in which patients with damage to visual cortical areas are able to accurately report on certain attributes of an object (e.g., its location) despite being unable to "see" the object consciously (Weiskrantz et al., 1974; Azzopardi and Cowey, 1997; Stoerig and Cowey, 1997). Reports of this striking dissociation between conscious awareness and high-level perceptual processes are almost exclusively confined to vision. However, there is evidence from two reports for an equivalent dissociation in touch. In the first case, a woman suffering damage to her left parietal cortex was able to identify with above-chance accuracy the location of tactile stimuli applied to her right hand or arm, although she could not detect the occurrence of those stimuli (Paillard et al., 1983). A similar dissociation was described by Rossetti et al. (1995) in their report of a patient with a left thalamic lesion. Such evidence would appear to reveal strict boundaries to conscious perception and show that some high-level perceptual processes capable of guiding complex behaviors are performed by neural systems that operate outside of conscious awareness.

If these neurological findings identify a basic feature of the

Received 0ct. 11, 2005; revised Dec. 7, 2005; accepted Dec. 8, 2005.

This work was supported by Australian Research Council Grant DP0343552.

Correspondence should be addressed to Justin Harris, School of Psychology, University of Sydney, Sydney 2006,

Australia. E-mail: justinh@psych.usyd.edu.au.

DOI:10.1523/JNEUROSCI.4318-05.2006

Copyright $\odot 2006$ Society for Neuroscience $\quad$ 0270-6474/06/260948-05\$15.00/0 organization of perceptual processes, it should be possible to demonstrate equivalent perceptual dissociations in neurologically intact "normal" subjects. We recently attempted to do this with touch, conducting careful psychophysical measurement of people's ability to detect a tactile stimulus and report its location (Harris et al., 2004). We found that detection and localization could be dissociated but that subjects were always better at detecting than localizing the stimulus. Moreover, based on detailed analysis of the response profiles for the two tasks, and comparison with simulated data generated by computational models, we concluded that the ability to localize the stimulus was dependent on detection, a conclusion inconsistent with the suggestion that localization can proceed in the absence of detection.

One potentially important difference between our psychophysical experiments and the previous neurological studies concerns the way that the subjects reported the location of the tactile stimulus. Our subjects named the finger on which the tactile stimulus was applied, whereas the neurological patients pointed at the location of the stimulus on their hand or arm. Thus, it is possible that the patients used implicit sensory information to guide their motor responses, information that was not available to our subjects when they made explicit verbal responses about the stimulus location. This interpretation is consistent with the proposed split between sensory processes for action versus conscious perception (Milner, 1995; Milner and Goodale, 1995; Rossetti et al., 1995). Here, we investigate this possibility with four psychophysical experiments with normal subjects who were required to report verbally whether they detected a tactile stimulus and to indicate its location with a simple motor response (pointing at or moving the stimulated finger). 
Table 1. Summary of experimental designs

\begin{tabular}{llll}
\hline & Detection task & Localization task & SOAs (ms) \\
\hline Experiment 1 & Yes-no & 4AFC point & $30,45,60,75$ \\
Experiment 2 & Yes-no & 4AFC move & $30,45,60,75$ \\
Experiment 3 & 2AFC when & 2AFC point & $30,60,90,120$ \\
Experiment 4 & 2AFC when & 2AFC move & $30,60,90,120$ \\
\hline
\end{tabular}

\section{Materials and Methods}

As in our previous experiments (Harris et al., 2004), the current experiments used a backward masking procedure to challenge our subjects' ability to detect and localize tactile stimuli so that we might observe a separation in performance between the two tasks. On each trial, subjects were presented with a brief tactile stimulus (the target) on one finger followed shortly afterward by a vibration (the mask) presented simultaneously to multiple fingers. The task was to say whether the target was present and to indicate on which finger. The temporal interval between the target and mask was varied to reveal systematic changes in detection and localization as the target became more salient. Four different stimulus onset asynchronies (SOAs) between the target and vibration were used in each experiment. In experiments 1 and 2, the SOAs were 30, 45, 60, and $75 \mathrm{~ms}$; in experiments 3 and 4, the SOAs were 30, 60, 90, and 120 $\mathrm{ms}$.

In experiments 1 and 2, the target was present on $80 \%$ of trials and could occur with equal probability on any of the four fingers of the right hand (excluding the thumb). The subjects gave a yes-no response to report whether they detected the target stimulus. They also gave a forcedchoice response about the location of the stimulus, even if they had reported that they did not detect it. In experiment 1 , they indicated the location of the stimulus by pointing at the relevant finger with their left index finger, a response similar to that performed by the neurological patients in the reported cases of "tactile blindsight." Experiment 2 was an exact replication of experiment 1 , except that the subjects indicated the location of the target by moving (raising) the relevant finger. We used this different response on the grounds that it may be more sensitive to any implicit sensory information about the location of the stimulus. In both experiments, all response-accuracy data were converted to a biasfree score $\left(d^{\prime}\right)$ so that comparison between detection and localization performance would not be confounded by any response bias that would affect the yes-no detection response but not the four-alternative forcedchoice (4AFC) localization response. In addition, in both experiments, we examined the subjects' localization accuracy conditional on their detection (i.e., we compared their accuracy in localizing the stimuli they detected vs those they missed).

As an alternative means to avoid the potential confound of a response bias when comparing yes-no and forced-choice tasks, experiments 3 and 4 used forced-choice tasks to assess both detection and localization. Each trial was divided into two temporal intervals, and the subjects were asked to report which interval contained the target (the target was always presented in one of the two intervals). Thus, on each trial, the subjects felt two masks, separated by $750 \mathrm{~ms}$, and both masks were presented on two fingers (index and middle). The target stimulus was presented to one of the two fingers before one of the two masks. For detection, the subjects reported when the target occurred (before the first or second mask); for localization, they indicated which finger received the target (index or middle). Experiments 3 and 4 differed only with respect to the localization response. The subjects either pointed at (experiment 3 ) or raised (experiment 4) the finger on which the target had been presented. A summary of the different experimental designs is presented in Table 1.

Experimental subjects. Forty first-year psychology students participated in the experiments for course credit. They were aged 18-22 years, 25 were female, and 36 were right handed. The recruitment of subjects and the experimental procedures had been approved by the institutional ethics committee.

Experimental apparatus. The target stimulus and vibration mask were produced using nickel bimorph wafers in an apparatus described previously (Harris et al., 2004). The target was a $5 \mathrm{~ms}$ upward square-wave deflection of $\sim 100 \mathrm{~mm}$. The mask was a $50 \mathrm{~Hz}$ square wave vibration of the same amplitude, lasting $500 \mathrm{~ms}$ in experiments 1 and 2 and 250 ms in experiments 3 and 4 (the mask was shortened because each trial included two masks).

Experimental procedure. The subjects sat with the four fingertips of their right hand (excluding the thumb) resting lightly on the buttons of the apparatus. The task was explained, and the subjects were familiarized with both the target stimulus and the mask. They then commenced a block of trials with different target-mask SOAs randomly intermixed (in one of four different randomized orders). On any trial, the target had equal probability of occurring on any of the four fingers in experiments 1 and 2 or on either the index or middle finger in experiments 3 and 4 . Experiments 1 and 2 included 20\% catch trials (target absent), randomly intermixed among the experimental trials, to determine each subject's false alarm rate for detection. In experiments 1 and 3, the subjects used their left index finger to point at the finger on which the target had been presented. To make sure each response could be recorded clearly by the experimenter, the subjects were asked to touch the chosen finger. In experiments 2 and 4 , the subjects moved (raised by $\sim 1 \mathrm{~cm}$ ) the finger that received the target stimulus.

\section{Results}

\section{Experiments 1 and 2}

Subjects ( $n=11$ in experiment $1 ; n=10$ in experiment 2 ) made detection and localization judgments on 400 trials ( 80 trials at each of the four SOAs plus 80 catch trials). Figure $1 A$ shows the mean accuracy for detection (as the percentage of targets that were detected) and localization (as the percentage of trials on which the subjects pointed at the correct finger) across each of the four target-mask SOAs in experiment 1 . Accuracy for both judgments increased as the SOA increased, but accuracy increased more steeply for detection than localization. Indeed, at very short SOAs, accuracy was higher for localization than detection. Essentially the same pattern is true for experiment 2 (Fig. $1 D$ ), in which subjects indicated the location of the target by moving the relevant finger. These results could be interpreted as showing that subjects are better at localizing than detecting targets at the shortest $\mathrm{SOA}$, in much the same way that the neurological patients described in the Introduction were able to localize correctly tactile stimuli that they failed to detect. However, this comparison between detection and localization accuracy is confounded because of the very different decision processes underlying each judgment.

In order to appropriately compare detection and localization accuracy, both were converted to $d^{\prime}$ scores, a bias-free measure of sensitivity, using the tables by Elliott (1964). For the detection task, $d^{\prime}$ was calculated using the false-alarm rate (the proportion of catch trials on which the subject incorrectly reported detecting a target). In experiments 1 and 2, the mean false alarm rates were 7.3 and $8.5 \%$, respectively, indicating a fairly conservative decision criterion. The mean $d^{\prime}$ scores for both detection and localization in the two experiments are shown in Figure 1, $B$ and $E$. It is clear that sensitivity was higher overall for detection than for localization in both experiments. Again, performance on both judgments increased as the SOA increased, but that the increase in accuracy was steeper for detection than localization. A repeated-measures ANOVA on the $d^{\prime}$ data confirmed these observations. There was a significant difference overall between detection and localization $\left(F_{(1,10)}=5.87, p=0.036\right.$ for experiment $1 ; F_{(1,9)}=22.41, p=0.001$ for experiment 2$)$; there was a significant linear trend across SOA $\left(F_{(1,10)}=44.93, p<0.001\right.$ for experiment $1 ; F_{(1,9)}=32.75, p<0.001$ for experiment 2$)$; and 


\section{Experiment 1}
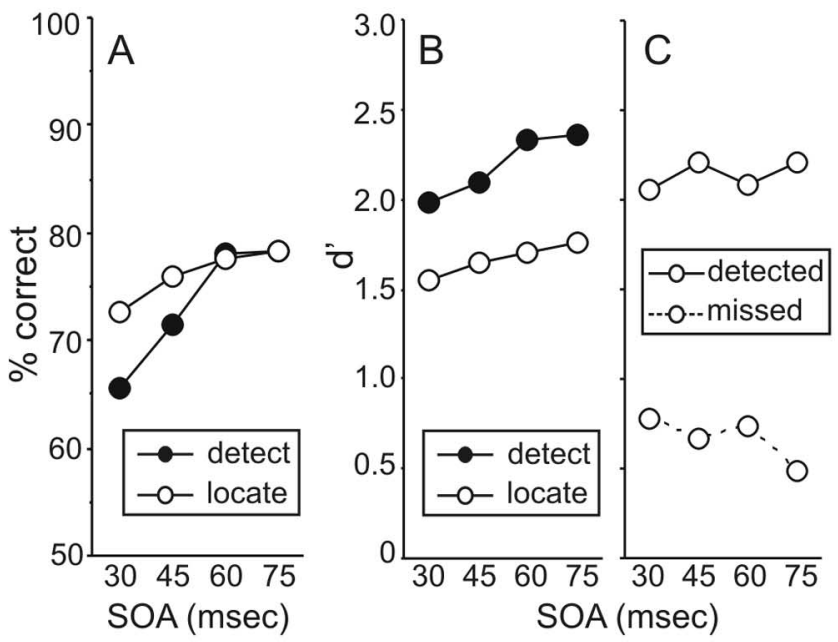

\section{Experiment 2}
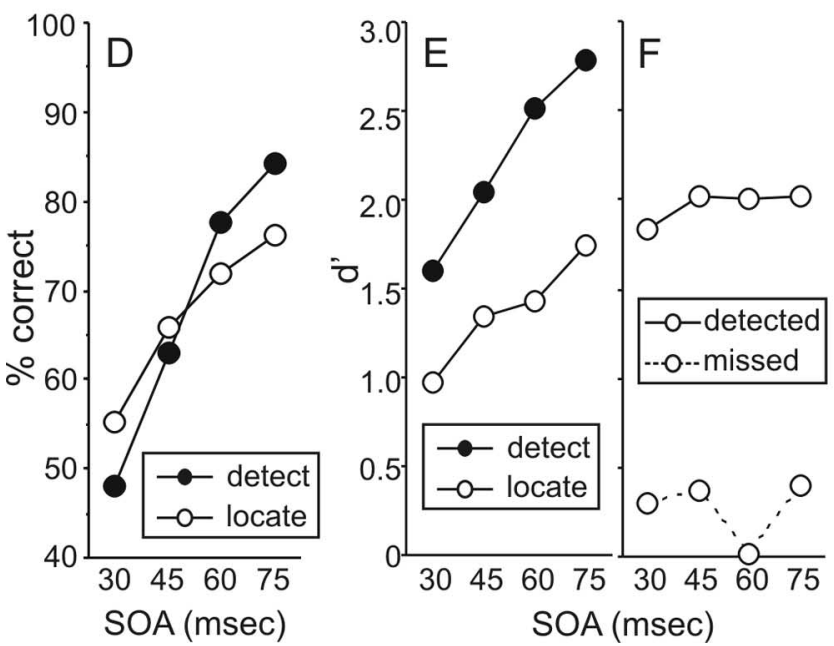

Figure 1. Results of experiment $1(\boldsymbol{A}-\boldsymbol{C})$ and experiment $2(\boldsymbol{D}-\boldsymbol{F})$. Subjects responded "yes"

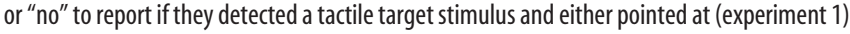
or moved (experiment 2) the relevant finger to indicate the location of the stimulus. $A, D$, The percentage correct for detection (hit rate) and localization (a 4AFC judgment). $\boldsymbol{B}$ and $\boldsymbol{E}$ show these scores converted to $d^{\prime}$ scores so that performance on the two tasks could be compared appropriately. Clearly, subjects were better at detecting than localizing the stimulus. C, $F$, Localization accuracy for trials on which the subjects detected the target versus trials when they missed the target. The subjects were much better at indicating the location of targets they detected than targets they missed, although in experiment $1(\boldsymbol{C})$, they performed better than chance at indicating the location of missed targets.

there was a significant interaction between task (detection vs localization $)$ and linear trend $\left(F_{(1,10)}=5.63, p=0.039\right.$ for experiment $1 ; F_{(1,9)}=8.57, p=0.017$ for experiment 2$)$.

The final analysis of these data compared the subjects' accuracy at localizing those targets they detected versus those they missed (Fig. 1C,F). The subjects were far more accurate at localizing the targets they detected than those they missed $\left(F_{(1,10)}=\right.$ 116.97, $p<0.001$ for experiment $1 ; F_{(1,9)}=74.86, p<0.001$ for experiment 2). Nonetheless, in experiment 1 , localization accuracy for missed targets was significantly above chance $\left(d^{\prime}=0\right)$ at each SOA $\left(t_{(10)}=4.01,2.84,4.30,2.41 ; p=0.002,0.018,0.002\right.$, $0.036)$. Localization accuracy for missed targets was lower in experiment 2 and was significantly above chance at only one SOA $\left(45 \mathrm{~ms} ; t_{(9)}=2.31 ; p=0.046\right)$. Finally, the localization accuracy for detected targets and missed targets did not change across SOA in either experiment $(F<1.0)$.

\section{Experiments 3 and 4}

Experiments 3 and 4 circumvented the problems associated with comparing yes-no detection decisions with 4AFC localization judgments by using a two-alternative choice (2AFC) task for both judgments. On each trial, subjects ( $n=10$ in experiment $3 ; n=9$ in experiment 4) felt two consecutive masks, $750 \mathrm{~ms}$ apart, each presented simultaneously to two fingers (index and middle). The target was presented on one of the two fingers immediately before one of the two masks. For detection, the subjects reported when the target occurred (before the first or second mask); for localization, they either pointed at (experiment 3 ) or moved (experiment 4) the targeted finger. In both experiments, the subjects made these judgments on 320 trials, 80 trials at each of four targetmask SOAs.

The mean accuracy (as percentage correct) for both detection and localization across each of the four SOAs is shown in Figure 2, $A$ and $C$. In experiment 3 , the subjects were slightly more accurate at detecting when the target occurred than pointing at the finger on which it occurred $\left(F_{(1,9)}=7.35 ; p=0.024\right)$. Both detection and localization accuracy increased with increasing $\operatorname{SOA}\left(F_{(1,9)}=\right.$ $102.18 ; p<0.001)$, and the increase in accuracy was steeper for detection than for localization $\left(F_{(1,9)}=9.66 ; p=0.012\right)$. In experiment 4 , both detection and localization accuracy increased with increasing $\operatorname{SOA}\left(F_{(1,8)}=49.16 ; p<0.001\right)$, but there were no differences between detection and localization $(F<1.0)$.

We compared localization accuracy between trials on which the subjects correctly reported when the stimulus occurred versus trials on which they named the wrong interval (i.e., they missed the target). In both experiments (Fig. $2 B, C$ ), there was a large difference between the two types of trial. The subjects were much more accurate if they had correctly reported when the stimulus occurred than if they had been incorrect $\left(F_{(1,9)}=35.28, p<0.001\right.$ for experiment $3 ; F_{(1,8)}=15.17, p=0.005$ for experiment 4$)$. Indeed, on trials when the subjects incorrectly reported the time of the target, they were at chance (50\% correct) in identifying its location. Finally, in both experiments, there was a clear linear trend across SOA for trials with correct detection responses $\left(F_{(1,9)}\right.$ $=34.42, p<0.001$ in experiment $3 ; F_{(1,8)}=13.18, p=0.007$ in experiment 4$)$, but there was no trend across SOA for trials with incorrect detection responses $\left(F_{(1,9)}<1\right.$ in experiment $3 ; F_{(1,8)}=$ 4.26, $p=0.073$ in experiment 4$)$.

\section{Discussion}

\section{Experiments 1 and 2}

These experiments show that subjects are more accurate at reporting the presence (vs absence) of a tactile stimulus than at indicating its location either by pointing at (experiment 1), or moving itself (experiment 2), the target finger. Both detection and localization accuracy improved as the SOA between the target and mask increased, but they improved at different rates; as the SOA increased, the subjects became increasingly more sensitive at detecting than localizing the stimulus. Finally, comparison of localization accuracy for detected targets versus missed targets revealed that detection and localization are correlated, because subjects were much more accurate at locating targets they had detected than targets they had missed. Nonetheless, in experiment 1 , the subjects were able to localize undetected targets with above-chance accuracy. This pattern of results is virtually identical to that we observed previously in similar experiments in 

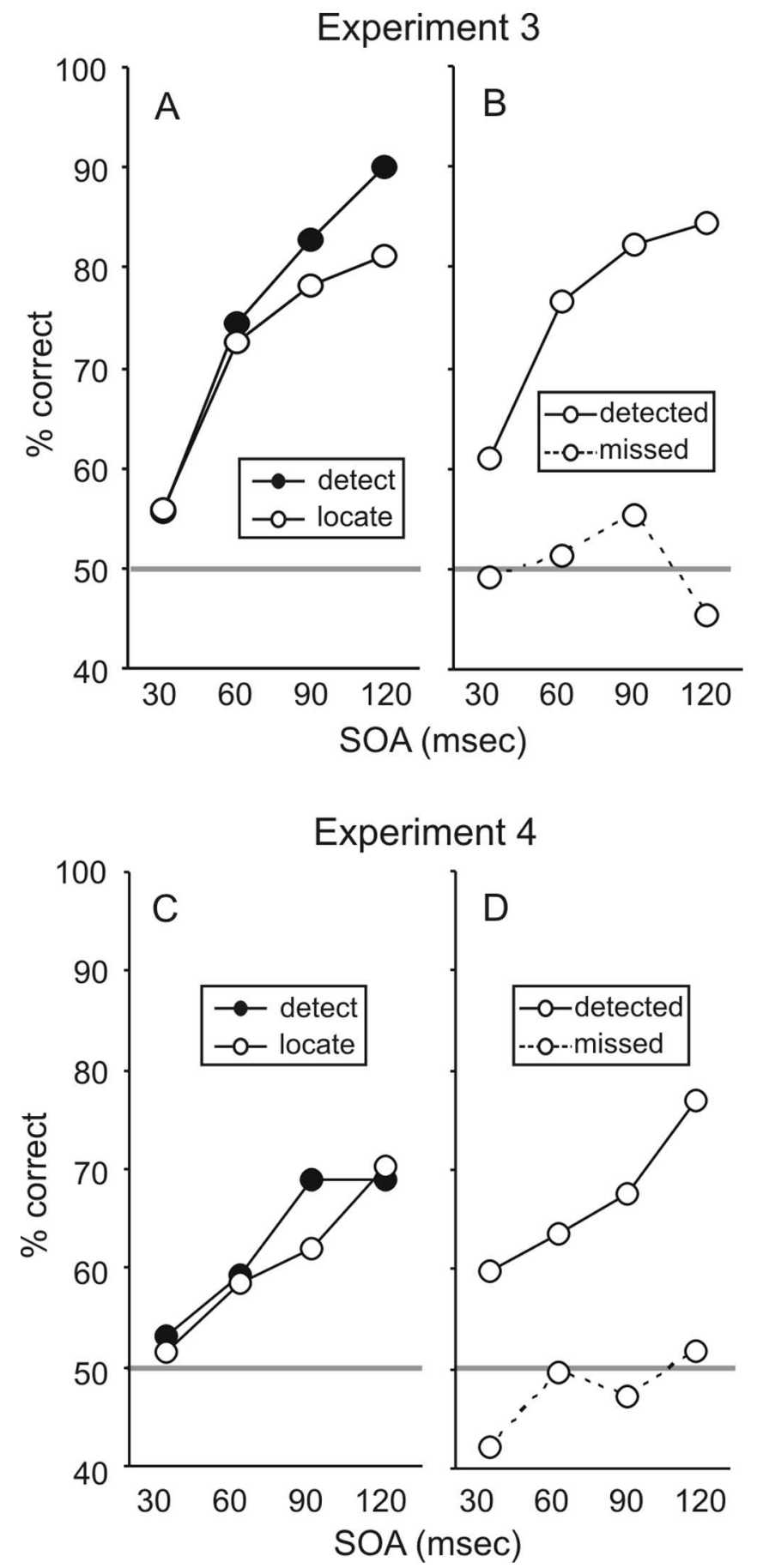

Figure 2. Results of experiment $3(\boldsymbol{A}, \boldsymbol{B})$ and experiment $4(\boldsymbol{C}, \boldsymbol{D})$. Subjects reported which of two temporal intervals contained a tactile stimulus (a 2AFC detection task) and either pointed at (experiment 3) or moved (experiment 4 ) the finger that received the target (a 2AFC localization task). $A, C$, The average percentage correct for detection and localization. Detection accuracy was slightly higher than localization accuracy in experiment 3 , but there was no difference in experiment 4. $\boldsymbol{B}, \boldsymbol{D}$, Localization accuracy for trials on which the subjects correctly identified when the target occurred versus trials on which they reported the wrong interval (i.e., they missed the target). The subjects were consistently at chance when attempting to indicate the location of missed targets.

which the subjects were required to identify the location of the target by naming the relevant finger (Harris et al., 2004). Therefore, the relationship between detection and localization of tactile stimuli does not change when subjects make motor responses rather than verbal responses to indicate the location of the stim- ulus. As argued in our previous paper, this pattern of results is most consistent with a sequential scheme in which the sensory processes leading to localization are subsequent to and dependent on the processes leading to detection (Harris et al., 2004).

\section{Experiments 3 and 4}

These experiments used matched 2AFC tasks to measure both detection and localization accuracy. In experiment 3, subjects were slightly more accurate at detecting when a tactile stimulus occurred than indicating its location by pointing at the stimulated finger. The subjects in experiment 4 were equally accurate at detecting when the stimulus occurred and indicating where it occurred by moving the stimulated finger. This correlation between detection and localization was underscored by the observation that subjects were relatively accurate at indicating the location of targets they had correctly detected but were at chance when trying to indicate the location of targets they had not detected. In other words, the subjects were completely unable to localize the tactile stimulus if they had failed to detect when it occurred. Finally, localization of detected targets improved across SOA, whereas localization of missed targets remained at chance. This indicates that improved localization with increased target-mask SOA is contingent on concurrent improvements in detection and that subjects cannot localize missed targets even at long SOAs.

\section{General discussion}

The four experiments presented here examined the relationship between the conscious detection of a tactile stimulus and the ability to identify its location by either pointing at or moving the stimulated finger. In three of the experiments, the subjects were more sensitive at detecting when the target occurred than identifying where it occurred. Additional evidence for this dissociation was provided by the finding that increments in the interval between the target and mask led to greater improvements in detection accuracy than localization accuracy. These differences indicate that detection is not sufficient for accurate localization and that localization is not necessary for detection.

Experiment 1 provided evidence that could be interpreted as revealing the reverse dissociation, that localization is not contingent on detection. Specifically, the subjects in this experiment performed significantly better than chance at locating stimuli that they failed to detect. Previously, we observed this trend in experiments in which subjects named the target finger (Harris et al., 2004). As such, both findings would appear to concur with neurological reports of patients who accurately indicated the location of tactile stimuli that they did not consciously detect (Paillard et al., 1983; Rossetti et al., 1995). However, as noted in our previous publication, this finding on its own does not constitute evidence of accurate localization in the absence of detection, because, in those experiments as well as the neurological studies, the subjects gave yes-no responses to report whether they detected the stimulus. This apparently simple response requires that subjects adopt a decision criterion. (They respond "yes" if their perception is above the criterion; otherwise, they respond "no."). In contrast, the forced choice used to measure localization ability does not require a criterion and is thus more sensitive to sensory inputs. As a result, the subjects would have been able to use whatever weak sensory signal was present to guide their forced-choice location judgments, even when that signal was below the decision criterion for detection. This confound would be particularly significant if the subjects adopted a conservative detection criterion, 
as appears to have been the case based on the low false-alarm rates recorded in the present experiments.

The possibility that subjects can localize a tactile stimulus independently of conscious detection is most directly addressed by the design used in experiments 3 and 4. Here, subjects made forced-choice judgments for both detection and localization (they reported which of two intervals the target was in and indicated which of two fingers it was on). Thus, both detection and localization were measured by equally sensitive tasks, and any difference in accuracy would reveal a true dissociation rather than a possible measurement artifact. Moreover, although subjects may correctly guess the timing of a target they did not detect in a forced-choice task, they would not incorrectly identify the timing of targets that they did detect (unless they failed to remember in which interval they detected the target, which is an unlikely event, given the minimal memory demand of the twoalternative choice). In other words, one can assume that a target was not detected if the subject reported it as occurring in the wrong interval. Therefore, it is significant that, in both experiments, the subjects performed at chance when attempting to indicate the location of missed targets, demonstrating the complete absence of localization in the absence of detection. We note that the when judgment used in experiments 3 and 4 may invoke a different mechanism from that underlying yes-no detection judgments, and thus it is possible, at least in principle, that when and where are associated in a way that detection and localization are not. However, this conclusion is contradicted by experiments 1 and 2, as well as our previous report (Harris et al., 2004), which used traditional yes-no detection and forced-choice localization tasks. In those experiments, the subjects' performance conformed better to a model in which localization is subsequent to, and dependent on, detection than to a model that assumes independence between these processes.

The dependence of localization on detection was confirmed by two additional findings obtained in all four experiments. First, there was a very large difference in localization accuracy between detected and missed targets, documenting a clear correlation between these processes. Second, localization accuracy for missed targets did not change across SOA, although localization accuracy in general improved as the target-mask SOA increased. Therefore, the general improvement in localization across SOA is related to the concurrent improvement in detection. Once this is factored out, there is no independent improvement in localization.

\section{Conclusions}

The present experiments confirmed that people's ability to identify the location of a tactile stimulus is contingent on conscious detection of that stimulus. The present experiments are particularly conclusive, because the subjects were completely unable to guess the location of the target stimulus if they had not correctly detected when it occurred. Thus, our findings are not consistent with previous reports of a blindsight-like syndrome in neurolog- ical patients who could accurately point to the location of tactile stimuli that they failed to detect (Paillard et al., 1983; Rossetti et al., 1995). Furthermore, the present experiments show that conscious detection is crucial for localization, even when subjects indicate the location of the stimulus by either pointing at or moving the stimulated finger. Thus, the discrepancy between our results and the neurological reports cannot be attributed to differences in the response used to assay localization, as might be implied by the proposed dissociation between sensory processes for conscious perception versus sensory processes for guiding action (Milner, 1995; Milner and Goodale, 1995; Rossetti et al., 1995). We suggest that the evidence provided by the previous neurological cases may be subject to the confound that affects many reports of visual blindsight (Campion et al., 1983), namely that the patient's ability to identify the location of a stimulus that they reported not detecting may be an artifact of the difference in the psychometric properties of the yes-no and forced-choice decisions used to index detection and localization, respectively [for the exception to this in a study of visual blindsight, see Azzopardi and Cowey (1997)]. Should future neurological investigations provide evidence of tactile blindsight that is not subject to this confound, that evidence would uncover an unconscious sensory process that, according to the present findings, cannot be readily isolated in the intact nervous system.

\section{References}

Azzopardi P, Cowey A (1997) Is blindsight like normal, near-threshold vision? Proc Natl Acad Sci USA 94:14190-14194.

Campion J, Latto R, Smith YM (1983) Is blindsight an effect of scattered light, spared cortex and near-threshold vision? Behav Brain Sci 6:423-486

Elliott PB (1964) Appendix 1-Tables of d'. In: Signal detection and recognition by human observers: contemporary readings (Swets JA, ed), pp 651-684. New York: Wiley.

Halligan PW, Hunt M, Marshall JC, Wade DT (1995) Sensory detection without localization. Neurocase 1:259-266.

Harris JA, Thein T, Clifford CWG (2004) Dissociating detection from localization of tactile stimuli. J Neurosci 24:3683-3693.

Head H, Holmes G (1911) Sensory disturbances from cerebral lesions. Brain 34:102-254.

Milner AD (1995) Cerebral correlates of visual awareness. Neuropsychologia 33:1117-1130.

Milner AD, Goodale MA (1995) The visual brain in action. Oxford: Oxford UP.

Paillard J, Michel F, Stelmach G (1983) Localization without content: a tactile analogue of 'blind sight'. Arch Neurol 40:548-551.

Rapp B, Hendel SK, Medina J (2002) Remodelling of somatosensory hand representations following cerebral lesions in humans. NeuroReport 13:207-211.

Rossetti Y, Rode G, Boisson D (1995) Implicit processing of somaesthetic information: a dissociation between where and how? NeuroReport 6:506-510.

Stoerig P, Cowey A (1997) Blindsight in man and monkey. Brain 120:535-559.

Weiskrantz L, Warrington EK, Sanders MD, Marshall JC (1974) Visual capacity in the hemianopic field following a restricted occipital ablation. Brain 97:709-728. 